$\S=-1$

\title{
Geometric-Gamma Collective Modified Value-at-Risk Model in Life Insurance Risk
}

\author{
${ }^{1}$ Muhammad Iqbal Al-Banna Ismail, Sukono, ${ }^{3}$ Abdul Talib BIN Bon, \\ ${ }^{4}$ Yuyun Hidayat, ${ }^{5}$ Eman Lesmana, and ${ }^{6}$ Sudradjat Supian \\ ${ }^{2,5,6}$ Department of Mathematics, Faculty of Mathematics and Natural Sciences, Universitas Padjadjaran, \\ Jl. Raya Bandung-Sumedang Km 21, Jatinangor 45363, Jawa Barat, Indonesia \\ ${ }^{4}$ Department of Statistics, Faculty of Mathematics and Natural Sciences, Universitas Padjadjaran, \\ Jl. Raya Bandung-Sumedang Km 21, Jatinangor 45363, Jawa Barat, Indonesia \\ ${ }^{3}$ Department of Production and Operation, Faculty of Technology Management and Business, \\ Universiti Tun Hessein Onn Malaysia, Batu Pahat, Johor, Malaysia \\ *Corresponding Author Email: gp150068@siswa.uthm.edu.my
}

\begin{abstract}
Claim risk is a payment made by the insurance company to the policyholder. Actuaries in insurance companies should be able to measure and control the risk of claims, in order to avoid losses to insurance companies. In this paper we analyze the Geometric-Gamma Collective Modified Value-at-Risk model in life insurance risk. In this research, there is a development of claim risk measure called Collective Modified Value-at-Risk, which is an extension of Collective Risk model. This Collective Modified Value-at-Risk model requires estimation of the mean, variance, skewness, and kurtosis parameters. The result of this research, is that the extent of this model can be applied to the risk of claims amount of non-normal distributed. Thus, the Collective Modified Value-at-Risk model can serve as one of the statistical alternatives for measuring the risk of claims on life insurance.
\end{abstract}

Keywords: Life insurance, claim risk, collective risk, Value-at-Risk, Collective Modified Value-at-Risk, non-normal distribution.

\section{Introduction}

Insurance can be seen from two points of view, namely first; As protection to the finances provided by the insurer, and secondly; As a risk pooling tool of two or more persons or companies through the promised donations to establish funds to pay claims [4]. Insurance is a means of risk transfer, requiring collective (agregate) risk, ie insurer combining risks from many insured. Through this collective risk insurer can improve the ability to predict expected losses [2]. Although most insurers collect enough pre-paid premiums to cover all of their expected losses, some of the insurers are unsure of part of the burden on the entire insured after the loss [5]. Therefore, the insurer considers it necessary to have the ability to measure risk of losses, which can minimize the risk level of these losses [12]. The magnitude of the need to measure the risk of losses more precisely, led to the need for many alternative measurement methods developed in the study ([9]; [8]).

Research on the risk measurement model of these losses has also been widely practiced by previous researchers. As for example, [1], examines the estimated total amount of claims in the auto insurance industry. This study aims to estimate insurance claims from a set of data using the Tweedie and zeroadjusted Gaussian inverse (ZAIG) methods. [3], examines the modeling of risk parameters in risk premiums in internal multi-year models. The study concludes that the risk parameters in the premium may have a significant impact on the company's risk situation, and are a risk that needs to be adequately considered. [11], conducts research on collective risk models for the distribution of claim reserves. In this study proposes a different way of analyzing the remarkable claims reserves based on the Collective Risk Theory approach. This collective risk is a combination of the frequency of claims and the amount of claims incurred. Related to the frequency of claims, Valecky (2016), has modeled the frequency of claims in motorcycle insurance, concluding that considering overdispersion is an important point in modeling the frequency of claims. [16], analyzing insurance claim data using Lognormal model. Meanwhile, [13] estimates the risk model of life insurance claims for cancer patients using the Bayesia method. The research of risk measurement model that has been done by the previous researcher above generally use approach of variance as risk measure. While it is worth noting, that the standard deviation or deviation is a measure of average risk, so it can not accommodate any risk events. Therefore, it is necessary to develop an alternative risk measurement model.

Based on the above description, this paper analyzes the development of Geometric-Gamma Collective Value-at-Risk and Modified Collective Value-at-Risk model. As for the object of research in this paper is life insurance claim data. Where the frequency of claims is assumed to follow the geometric distribution model, and the claim amount is assumed to follow the Gamma distribution model. The purpose of this study is to develop an alternative risk measurement model, to measure the risk of aggregate losses on life insurance claims. 


\section{Problem Statement}

Insurance is a tools in handling risk which is done by transferring from one party to another party which in this case will be the insurance company. Economically, insurance means collecting fund which can be use to close or giving compensation to the person who is the one having the loss. Insurance is a business taking over the risk from customer to the insurance company until the customer feels comfortable to follow the insurance program. Insurance must be good to handle the risk so the business will be profitable so the customer feels comfortable to follow the offering program. In insurance, experience about risk is the occurrence of the insured claim. Risk is generally been measure by variance and standard deviation. But should be notice that variance and standard deviation is an average risk size, which it could not accommodate all of risk until it be need to find other alternative.

One alternative to measure risk is using Value at Risk. Value at Risk (VaR) is one way to answer by measuring risk probability can be experienced by a claim. VaR is a measurement can be use to asses the worst loss which it probably can happen to an insurance company, weather its individual or in aggregate (collective) in one time, at level chance set. In VaR, probably of loss is calculated by probability loss is worst from a set percentage. VaR is a quantile measurement which been assume that function probability is following the normal distribution. The problem is how if the function probability is not following the normal distribution. That is why it is needed a modification from Value at Risk which is known as Modified Value at Risk (MVaR). The Modified VaR is applied in AlternativeSoft's platform. The skewness and the kurtosis effect is high if the $\mathrm{VaR}$ is computed at 99\% (Sukono et all., 2016).

In one insurance system the risk is happen from the insured can bring up claim. Claim is a compensation for a risk loss. Individual claim in one period insurance is called aggregation claim while aggregation claim is collective risk [2]. Should be know that collective risk is calculate based on variance and standard deviation. As mention above variance and standard deviation could not accommodate all risk event. That is why, in this research we will develop risk measure base on Collective Value at Risk $(\mathrm{CVaR})$ and Collective Modified Value at Risk (CMVaR).

\section{Materials and Methods}

This section discusses the materials and methods used in the research in this paper. In the methods used, discussed include: claims distribution model, collective risk model, Collective Valueat-Risk model, and Collective Modified Value-at-Risk model.

\subsection{Materials}

The data used in the research in the development of Collective Value-at-Risk $(\mathrm{CVaR})$ model, and Collective Modified Value-atRisk $(C M V a R)$ model, is the claim data from $\mathrm{ABC}$ insurance company. These claims data are secondary data, which can be grouped into two types, namely claims frequency and claim amount. Claim data from ABC insurance company during 2016, summarizing the frequency of claims occurring as many as 91 events, and the claim amount reaches IDR 221,639,944.00.

\subsection{Methods}

This section discusses the models used in the analysis methods in this paper. The discussion includes: the claim distribution model, the collective risk model, the collective Value-at-Risk model, and the Collective Modified Value-at-Risk model.

\subsection{Claim Distribution Model}

This section discusses the distribution of claims, which consists of the distribution of claim frequency and the distribution of claim amount. In this paper the claim frequency is assumed to follow the geometric distribution model. So if we let $N$ isthe random variable of the claim frequency with the value $n$, and $p$ is the probability of occurrence

parameter a claim, the claim is the function of the probability of occurrence (Dicson, 2005; Valecky, 2016)

$$
\eta(n, p)=\left\{\begin{array}{c}
p q^{n-1} ; n=1,2,3, \ldots \\
0 ; \quad n \text { otherwishe }
\end{array}\right.
$$

where $q=1-p$ is a parameter of claim does not happen. The moment generating function of equation (1) is shaped ([8]; [5]):

$$
M_{N}(t)=\frac{p e^{t}}{\left(1-q e^{t}\right)} \text {. }
$$

While the claim amount, in this paper is assumed to follow the distribution of Gamma. Thus, if we let $X$ random variable of claim amount with value $x$, as well $\alpha$ and $\beta$ are the Gamma distribution parameters, then the probability density distribution function of kalim amount is ([2]; [13]):

$$
f(x)=\left\{\begin{array}{c}
\frac{1}{\Gamma(\alpha) \beta^{\alpha}} x^{n-1} e^{-x / \beta} ; x>0 \\
0 ; x \text { otherwishe }
\end{array},\right.
$$

Moment generating function to $k$ from equation (3) are:

$$
m_{k}=M_{X}(k)=(1-\beta k)^{-\alpha} ; k=1,2,3,4 \text {. }
$$

Collective Risk Model. If we let $S$ is a random variable of the aggregate claims amount, $N$ random variable of claims frequency, and $X_{i}$ random variables of claim amount of individuals $i$, the aggregate of the claims expressed as [11]:

$$
S=\sum_{i=1}^{N} X_{i}
$$

So that the conditional expectation of equation (4) is $E[S \mid N=n]=E\left[\sum_{i=1}^{N} X_{i}\right]=\sum_{i=1}^{N} E\left[X_{i}\right]=n m_{1} \quad, \quad$ and for $n=0,1,2 \ldots ., E[S \mid N]=N m_{1}$, so that the unconditional expectation of (4) is:

$E[S]=E\left[N m_{1}\right]=E[N] m_{1}$.

While the conditional variance of equation (4) is $\operatorname{Var}[S \mid N=n]=\operatorname{Var}\left[\sum_{i=1}^{N} X_{i}\right]=\sum_{i=1}^{N} \operatorname{Var}\left[X_{i}\right]=n\left(m_{2}-m_{1}^{2}\right)$, and for $n=0,1,2 \ldots ., \operatorname{Var}[S \mid N]=N\left(m_{2}-m_{1}^{2}\right)$. Therefore, the unconditional variance of (4) is: 


$$
\begin{aligned}
& \operatorname{Var}[S]=E[\operatorname{Var}(S \mid N)]+\operatorname{Var}[E(S \mid N)]= \\
& E[N]\left(m_{2}-m_{1}^{2}\right)+\operatorname{Var}[N] m_{1}^{2} .
\end{aligned}
$$

Furthermore, if it is assumed that $\left\{X_{i}\right\}_{i=1}^{\infty}$ independent and identically distributed (iid), then the moment function of equation (4) can be expressed as [2]:$$
M_{S}(t)=E\left[\left\{M_{X}(t)\right\}^{N}\right]=E\left[\exp \left\{\log \left\{M_{X}(t)\right\}^{N}\right]=M_{N}(t)\right.
$$ \\ 3.4 Models of Collective Value-at-Risk and Collective Modified Value-at-Risk}

Referring to [6] and [15], Value-at-Risk $(\mathrm{VaR})$ is a measure of market risk, which means when invested in initial capital $V_{0}$, in asset markets that have normal distribution with a mean return $\mu$ and standard deviation $\sigma$,

will have the potential maximum loss is equal to $\operatorname{VaR}=-V_{0}\left(\mu+z_{c} \sigma\right)$, where $z_{c}$ percentile of the standard normal distribution at a significance level $(1-c) \%$. If the principles of Value-at-Risk expanded its application as an alternative to aggregate claims risk measurement equation (4), it can be formulated so-called Collective Value-at-Risk $(\mathrm{CVaR})$ as:

$$
\begin{aligned}
& C \operatorname{VaR}=-\tilde{N}_{0}\left(E[S]+z_{c}\{\operatorname{Var}[S]\}^{1 / 2}\right)= \\
& -\tilde{N}_{0}\left(E[N] m_{1}+z_{c}\left\{E[N]\left(m_{2}-m_{1}^{2}\right)+\operatorname{Var}[N] m_{1}^{2}\right\}^{1 / 2}\right) .
\end{aligned}
$$

That is, if within a period of time there are as many claims qrequency $\tilde{N}_{0}$, which has a mean aggregate claims as equation (5) and the variance as equation (6), will have the risk of loss of the equation (8), where $z_{c}$ percentile of the standard normal distribution at a significance level $(1-c) \%$.

Meanwhile, if the return of market assets is non-normally distributed, according to [6] and Guarda et al. (2012), then potential losses can be measured using Modified Value-at-Risk $(M V a R)$. Thus, if this Modified Value-at-Risk principle is extended as an alternative to the risk of aggregate claims of equation (4) that is not normally distributed, then it can be formulated as Collective Modified Value-at-Risk ( $C M V a R)$ as:

$$
C M V a R=\tilde{N}_{0}\left\{E[S]+\left(z_{c}+\frac{1}{6}\left(z_{c}^{2}-1\right) \varsigma[S]+\frac{1}{12}\left(z_{c}^{3}-3 z_{c}\right) \kappa[S]-\frac{1}{36}\left(2 z_{c}^{3}-5 z_{c}\right)(\varsigma[S])^{2}\right)(\operatorname{Var}[S])^{1 / 2}\right\},
$$

where $E[S], \operatorname{Var}[S], \varsigma[S]$, and $\kappa[S]$, respectively are mean, variance, skewness, and kurtosis of aggregate claim as equation (4). Parameters of $\varsigma[S]$ and $\kappa[S]$, specifically require the use of successive third and fourth moments of the aggregate claims distribution equation (4).

\section{Results and Discussion}

This section describes the results and discussion, covering the description of the results of data processing analysis, and a description of the usefulness of each value obtained from the analysis of data processing.

\subsection{Results}

In this section we explore the results of the analysis of data processing, which includes: estimation of the claim frequency distribution model, estimation of the distribution model of the claim amount, and estimates the collective risk of insurance claims.

Estimation of the distribution model of the claims frequency. The estimation of the distribution model of the claims frequency is made by referring to equation (1), with the following stages: identification of the distribution model, distribution model parameter estimation, and distribution model matching test. The claims frequency occurring is a random sequence of events that can be predicted by Discrete distribution models. Identify the distribution model of claims frequency made through curve matching, using software of EasyFit 5.5. The result of matching this curve, indicating that the model suitable for claim frequency is following Geometric distribution. While estimation of distribution model of claim frequency is done by using Maximum Likelihood Estimator method. Estimates were made using software of EasyFit 5.5, and obtained the parameter estimator $p=0.1165$. Furthermore, the parameter estimator needs to be tested for suitability, based on predetermined assumptions. This distribution fit test was performed using Kolmogorov-Smirnov statistics. The hypothesis tested is $H_{0}$ : claims frequency data follow the Geometric distribution, against the alternative $H_{1}$ : claims frequency data does not follow the Geometric distribution. Kolmogorov-Smirnov test results, obtained the test statistics $D=$ 0.29504 . While at $95 \%$ confidence level, obtained critical value $D_{1-95 \%}=0.37543$. Because $D<D_{1-95 \%}$, it is clear that $H_{0}$ be accepted. This means that the claim frequency data follows the Geometric distribution, with the probability density function for Geometric distribution as follows:

$\eta(n, 0.1165)=(0.1165)(1-0.1165)^{n-1} ; n=1,2,3, \ldots$

The distribution model estimate of this claim frequency, then, is used to estimate the collective risk of insurance claims.

Estimation of the distribution model of the claim amount. The estimation of the distribution model of the claim amount is done by referring to equation (3), with the following stages: model identification, model parameter estimation, and model fit test. Identification of a distribution model of individual claim amount, made by matching the histogram distribution function. This curve matching is done using software of EasyFit 5.5. The result of the matching curve, obtained by a suitable distribution for individual claim amount data, is the Gamma distribution. Furthermore, the obtained distribution is estimated parameter with Maximum Likelihood Estimation method. Estimator value obtained is $\alpha=$ 152.71 and $\beta=0.08352$. Further, testing the suitability of the distribution based on the assumptions that had been predetermined. This distribution fit test was performed using Kolmogorov-Smirnov statistics. The hypothesis tested is $H_{0}$ : claims amount data follows the Gamma distribution, against alternatives $H_{1}$ : claim amount data do not follow the Gamma distribution. Kolmogorov-Smirnov test results obtained the test statistics $D=0.10341$. While at $95 \%$ confidence level, obtained critical value $D_{1-95 \%}=0.37543$. Because $D<D_{1-95 \%}$, it is 
clear that $H_{0}$ be accepted. Means that claim amount data follows the Gamma distribution

with an probability density function for Gamma distribution as follows:

$$
f(x)=\frac{1}{\Gamma(152.71)(0.08352)^{152.71}} x^{152.71-1} e^{-(x / 0.08352)}, x>0 .
$$

This distribution model estimator of claims amount, together with the distribution model estimator of claim frequency, is then used for estimating collective risk.

Estimation of the collective risk of insurance claims. This section discusses the collective risk estimates of insurance claims. The discussion covers the expectations of aggregate claims and the measurement of collective risk. Collective risk measurements are based on: Collective Risk (standard), Collective Value-at-Risk, and Collective Modified Value-at-Risk. Estimated expectations of aggregate claims and collective risk measurement, done based on claim frequency estimator distribution model equation (10), and the distribution model of the claim amount estimator equation (11). In estimating aggregate claim expectations and collective risk measurements, estimators of parameter values are required: $E[N]$ and $\operatorname{Var}[N]$, as well as the value of moments estimator of: $m_{1}, m_{2}, m_{3}$, and $m_{4}$.

Using the distribution model estimator of the claim frequency of equation (1), an estimator of the parameter value can be obtained

$$
\operatorname{CVaR}=-91\left\{-2.3263\left(3.2275 \times 10^{13}\right)^{1 / 1}\right.
$$

If the risk of aggregate claims is measured by Collective Modified Value-at-Risk (CMVaR), it is necessary to determine the estimators of Skewness $\varsigma[S]$ and Kurtosis $\kappa[S]$. Using the estimator of parameter values: $p, m_{1}, m_{2}, m_{3}$ and $m_{4}$, can be obtained the values of skewness $\varsigma[S]=3.587486$, and kurtosis $\kappa[S]=15.12596771$. Collective Modified Value-at-Risk $(C M V a R)$, on the assumption that the significance level $c=0.01$, and the claims frequency in one year is $\tilde{N}_{0}=91$, can be determined using equation (9). Based on calculations obtained the magnitude of $C M V a R$ is $C M V a R=51589567$.

\subsection{Discussion}

Claim data from the $\mathrm{ABC}$ insurance company during the year 2016, the incidence of each month is fluctuating both the frequency and amount of the claim. As has been explained, briefly the claims frequency occurred as much as 91 events, and the claims amount reached IDR 221,639,944.00. Meanwhile, based on the analysis results obtained the expected value of aggregate claim $E[S]$, its value is equal to $\mu_{S}=$ IDR $5,222,422.13$ per month or IDR $62,669,066.00$ a year. Where risks are measured by base Collective Risk (Variance of agregate claim) $\operatorname{Var}[S]$, its value is equal to $\sigma_{S}^{2}=3.2275 \times 10^{13}$ or $\sigma_{S}=5,681,109.00$ per month or IDR 68,173,309.00 a year.

If the risk of aggregate claims is measured by the Collective Value-at-Risk $(C V a R)$ model at the significance level $=0.01$, and assuming the claim frequency within a year is $\tilde{N}_{0}=91$, the obtained estimator of Collective Value-at-Risk ( $C V a R)$ of IDR $727,379,339.13$. Whereas, when the risk of aggregate claims is measured using the Collective Modified Value-at-Risk (CMVaR) model, at the same level of significance and assumption of the
$E[N]=8.584$ and estimator parameter value $\operatorname{Var}[N]=$ 65.100. While using the estimator of the distribution model of the equation of claim amount of equation (11),

an estimator of the values of moments can be obtained: $m_{1}=608412.178, m_{2}=1.32278 \times 10^{12}$;

$m_{3}=1.3456 \times 10^{19}$; and $m_{4}=9.23033 \times 10^{26}$.

Expected value of agregate claim $E[S]$, determined with reference to the equation (5), and the result is that $\mu_{S}=E[S]=5222422.13$.

While Collective Risk (Variance of agregate claim) $\operatorname{Var}[S]$, determined by reference to the equation (5), and the result is that $\sigma_{S}^{2}=\operatorname{Var}[S]=3.2275 \times 10^{13}$.

Furthermore, if the risk of aggregate claims is measured by Collective Value-at-Risk $(\mathrm{CVaR})$ at the level of significance $c=$ 0.01 , then obtained a standard normal distribution percentiles $z_{0.01}=-2.3263$.

Suppose the claim frequency within a year is $\tilde{N}_{0}=91$, value of Collective Value-at-Risk $(\mathrm{CVaR})$ can be calculated using equation (8) as follows:
$1 / 2+5222422.13\}=727379339.8$

claim frequency, it is obtained Collective Modified Value-at-Risk (CMVaR) of IDR 515,895,670.00.

Which size of risk, in this case that will be applied in the ABC insurance company, certainly depends on several considerations, both quantitative and qualitative. At least, the analysis of the development of this aggregate claim risk measurement model can serve as an alternative. Because measurement of risk is very important with regard to the determination of a reasonable aggregate premium, meaning it does not harm the insurance company but is covered by the insured.

\section{Conclusion}

In this paper we have analyzed the Geometric-Gamma Collective Modified Value-at-Risk model in life insurance risk. The data analyzed in this paper is secondary data obtained from the ABC insurance company. Based on data analysis, it can be concluded as follows: the claims frequency follows the Geometric distribution model with the parameter estimator $\hat{p}=0.1165$. Meanwhile, the claim amount following the model of Gamma distribution with parameter estimator $\hat{\alpha}=152.71$ and $\hat{\beta}=0.08352$. Thus, the aggregate claim model formed from the distributions of the claims frequency and the individual claim amount, is a geometric compound distribution. Based on the distribution of geometric compound formed moments $m_{1}=608412.178 ; m_{2}=$ $1.32278 \times 10^{12} ; \quad m_{3}=1.34565 \times 10^{19} ;$ and $m_{4}=9.23033 \times 10^{26}$. Using values of $\hat{p}, m_{1}, m_{2}, m_{3}$, and $m_{4}$, obtained estimator values: mean $\hat{\mu}_{S}=5222422.13$; variance $\hat{\sigma}_{S}^{2}=3.2275 \times 10^{13}$; skewness $\hat{\varsigma}_{S}=3.587486$; and kurtosis $\hat{\kappa}_{S}=15.12596771$. Thus, with a significance level of $5 \%$ obtained Collective Modified Value-at-Risk (CMVaR) of IDR 515,895,670.00. This value is a 
measure of alternative risk, especially if the insurance claim does not follow the normal distribution.

\section{Acknowledgments}

Further thanks to the Rector, Director of DRPMI, and Dean of FMIPA, Universitas Padjadjaran, which has a grant program of the Academic Leadership Grant (ALG) under the coordination of Prof. Dr. Sudradjat, and a grant program of Competence Research of Lecturer of Unpad (Riset Kompetensi Dosen Unpad/RKDU) under the coordination of Dr. Sukono, which is a means to increase research activities and publications to researchers at the Universitas Padjadjaran.

\section{References}

[1] Bortoluzzo, A.B., D. P. Claro, M. A. L. Caetano, R. Artes, 2011 Estimating Total Claim Size in the Auto Insurance Industry: a Comparison between Tweedie and Zero-Adjusted Inverse Gaussian Distribution, BAR, Curitiba, v. 8, n. 1, art. 3, pp. 37-47, Jan./Mar. 2011, Available online at, http://www.anpad.org.br/bar.

[2] Dickson, D.C.M. 2005. Insurance Risk and Ruin. Cambridge : Cambridge University Press.

[3] Diers, D., M.A. Eling, and M.A. Linde, 2012, Modeling Parameter Risk in Premium Risk in Multi-Year Internal Models, Working Papers on Risk Management and Insurance NO. 119, November 2012, pp. 1-25.

[4] Dionne, G. 2013, Risk Management: History, Difinition and Critique, Cirrelt-2013-17, March 2013, pp. 1-22. www.cirrelt.ca.

[5] Djuric, Z., 2013, Collective Risk Model in Non-Life Insurance, Economic Horizons, May - August 2013, Volume 15, Number 2, pp. $167-175$, UDC: 33 eISSN 2217-9232 , UDC: 005.334:368.025.6 ; 347.426.6, doi: 10.5937/ekonhor1302163D.

[6] Dowd, K. 2002. An Introduction to Market Risk Measurement, John Wiley \& Sons, Inc., New Delhi, India.

[7] Guarda, P., A. Rouabah, and J. Theal, 2012, An MVaR Framework to Capture Extreme Events in Macro-Prudential Stress Tests, Working Paper Series, NO 1464 / AUGUST 2012, pp. 1-46.

[8] Klugman, S.A., H.H. Panjer, and G.E. Willmot, 1998, Loss Models: From Data to Decision, Wiley Series in Probability and Statistics, John Wiley \& Sons, Inc., New York.

[9] Kolkovska, E.T., 2011, Risk Measures for Classical and Perturbed Risk Processes - A Survey, Pliska Stud. Math. Bulgar. 20 (2011), pp. 121-134.

[10] Kutub, U.M., I.M. Rafiqul, and R. Taslima, 2011, Mathematical Modeling of Life Insurance Policies, European Journal of Business and Management, ISSN 2222-1905 (Paper) ISSN 2222-2839 (Online), Vol 3, No.4, 2011, pp. 308-321. www.iiste.org.

[11] Nino, S. and C.G. Paolo, 2010, A Collective Risk Model for Claims Reserve Distribution, 29 th International Congress of Actuaries ICA 2010, Cape Town - March 7-12th 2010, pp. 1-22.

[12] Polanski, A., E. Stoja, and R. Zhang, 2013, Multidimensional Risk and Risk Dependence, Paper, pp. 1-38, University of East Anglia, Norwich Research Park, Norwich, NR4 7TJ, UK. Email: A.Polanski@uea.ac.uk.

[13] Sukono, Suyudi, M., Islamiyati, F., and Supian, S. 2017. Estimation Model of Life Insurance Claims Risk for Cancer Patients by Using Bayesian Method. IOP Conf. Series: Materials Science and

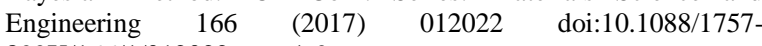
899X/166/1/012022. pp. $1-9$.

[14] Valecký, J., 2016, Modelling Claim Frequency in Vehicle Insurance, ACTA Universitatis Agriculturae Et Silviculturae Mendelianae Brunensis, Volume 64, Number 2, 2016, pp. 683-689. http://dx.doi.org/10.11118/ actaun201664020683.

[15] Yildirim, I., 2015, Financial Risk Measurement for Turkish Insurance Companies Using VaR Models, Journal of Financial Risk Management, 2015, 4, pp. 158-167, Published Online September 2015 in SciRes. http://www.scirp.org/journal/jfrm. http://dx.doi.org/10.4236/jfrm.2015.43013.

[16] Zuanetti, D.A., C.A.R. Diniz, and J.G. Leite, 2006, A Lognormal Model for Insurance Claims Data, REVSTAT - Statistical Journal, Volume 4, Number 2, June 2006, pp. 131-142. 